\title{
How regular is a regular-interval timetable? From theory to application
}

\author{
P. Tzieropoulos, D. Emery \& D. Tron \\ Group EPFL-LITEP - Intermodality and Transport Planning École, \\ Polytechnique Fédérale de Lausanne, Switzerland
}

\begin{abstract}
Initially, choice of regular-interval timetable was mostly addressing operational concerns, aiming to increase the network throughput and to smooth the day-today tasks of the personnel. Separation between infrastructure management and train operations, induced by the European Union since the early $90 \mathrm{~s}$, and the future opening of the rail services to competition, pushes more and more infrastructure managers to operate their network with regular-interval timetable. Thus, the interest of measuring the degree of regularity.

The paper defines the different steps needed for going from conventional operations to fully coordinated regular-interval timetable (the so-called clockface timetable). It starts by defining the basic notions, and shows some fundamental properties of regular-based timetables. Then, based on the definitions, a methodology is developed to measure and assess the regularity of a timetable, for a line and over a full-scale network. This is because, in practice, implementation of a perfectly regular timetable is not possible and, perhaps, neither desirable. Constraints related to demand or to resources lead to cancel train paths during off-peak periods or to provide extra stops or longer dwell times (and thus slowing down travel time) during peak hours, for instance.

More specifically, the paper presents a methodology for determining the interval used to evaluate and compare reference and actual timetables, per train class and by corridors. Tolerances in measuring are dealt with. The developed methodology has been used to develop assessment software, which has been used in a real life application.
\end{abstract}

Keywords: regular-interval timetable, coordinated cycling timetable. 


\section{Introduction}

A regular-interval timetable provides identical train paths for each service, scheduled at regular time intervals. A coordinated, or so-called clock-faced timetable [3], is based on the same principle and adds to it scheduled and guaranteed connections in selected main stations.

Nowadays, several European countries operate their train services on the basis of a regular-interval timetable. Those who do not yet, are gradually coming to this type of operation, too. Initially, the choice of regular-interval timetable was mostly addressing operational concerns. Systematic operations help both increasing the network throughput, and smoothing the day-to-day tasks of the personnel. Separation between infrastructure management and train operations, induced by the European Union since the early 90 s, and the ongoing opening of the rail services to competition, pushes more and more infrastructure managers to operate their network on regular-interval timetable.

Even based on a regular-interval principle, a timetable almost never strictly adheres to this principle. Early morning and late night services usually diverge from the standard train path design. Reinforcement train paths are often necessary during peak periods. Cost concerns may lead train operators to alleviate off-peak service by cancelling some train paths. Finally, especially in suburban and regional services, political pressures may also generate some diversions from the standard train path service by imposing extra stops.

Transgressions of the regular-interval pattern may negate (and often do) the main expected advantages from the regularity. To actually assess the cost of those transgressions, one needs to go for a detailed analysis and comparison of the actual timetable against a perfectly "orthodox" one. This is a cumbersome process that, to the knowledge of these authors, has never been conducted. In order to help planners and transport authorities to proceed with an initial fast assessment of the regularity of a timetable, an evaluation methodology has been developed and implemented as a software package [5]. The developed software has been applied to the French Rhône-Alpes Region, which in 2008 rescheduled its regional services on a regular-interval basis [6].

To design the methodology and to develop the software, it was first necessary to specify precisely the notions of structure, regularity and connectivity. This was done by referring back to the theory of regular-interval timetabling, and by developing specific notions as needed in the process. The paper sets the theoretical framework of regular-interval timetables, shows the fundamental properties of the latter, presents the options taken for measuring the regularity, and highlights the advantages and drawbacks of the methodology.

\section{Definitions}

Urban services have been operated with constant headings almost since their beginning. Often, this has also been the case of shuttle services. Dutch railways have been probably the first to apply this principle at the scale of the national network services in the late 1940s. It was called rigid timetable, by then. Some 
European networks came to the same principle during the last quarter of the $20^{\text {th }}$ century. They opted for it in order to both achieve productivity gains by systematising their operations, and to offer full time coverage of the services, much alike the car which is available for a journey at any time of the day. By sending the message to the customers that train services are also available at any time during the day, railways aim to enhance their competitive stance.

Some basic definitions are needed here to set the scenery. The first is the one of service, kind of product mould for the operator. In this context, a service is composed by [6]:

- a directional path in the network (defined by its origin, destination, and route),

- a stopping pattern (defining the intermediate stops and their duration),

- a commercial identity, which may be related with

○ travel time objectives,

- choice of rolling stock assigned to this particular mission,

$\circ$ fare policy,

○ package of extra services, etc.

Usually, any given service has its dual one, the return path.

A structured timetable is the one that keeps the service typology under control [6]:

- with a finite (and not too large) number of services, to ensure that the transport supply remains readable for customers and operators as well;

- with fairly distinct services, that are easily identifiable; supplying a range of products that are easy to identify makes consumer choices simple (and helps improving the marketing, too);

- with each particular train assigned to a given service (by avoiding planning "outlier" trains, that are hard to recognize by both customers and operators and which degrade the readability of the whole transport supply).

With a structured timetable, customers still need consulting the timetable, though they can easily identify local, fast, high-speed trains, etc.

A regular-interval timetable is a structured one and, what is more, with successive identical services planned at fixed time intervals [6]; services are periodical, and the time interval is the period. Theoretically, periodicity may not be the same for various services although, to fully benefit from the systematic properties, periods are usually unique or integer multiples of a basic time interval. Theoretically too, the time interval may be of any value and, for independently optimised shuttle services, it reflects the round trip time on the route, or - depending on supply level requirements - a multiplier or an integer fraction of it (Figure 1). However, for a network with interconnected lines, there is a strong impetus to opt for a unique time interval, often set to a round value, e.g. 60 minutes. In this case, customers only need to remember the departure minute of their usual service: if it is 12 , for instance, for a fast train leaving town A for town B, they know this same service is available at 7:12, 8:12, 9:12, and so on.

A coordinated regular timetable (or clock-faced timetable) is a regularinterval timetable that fulfils three additional constraints [6]: 


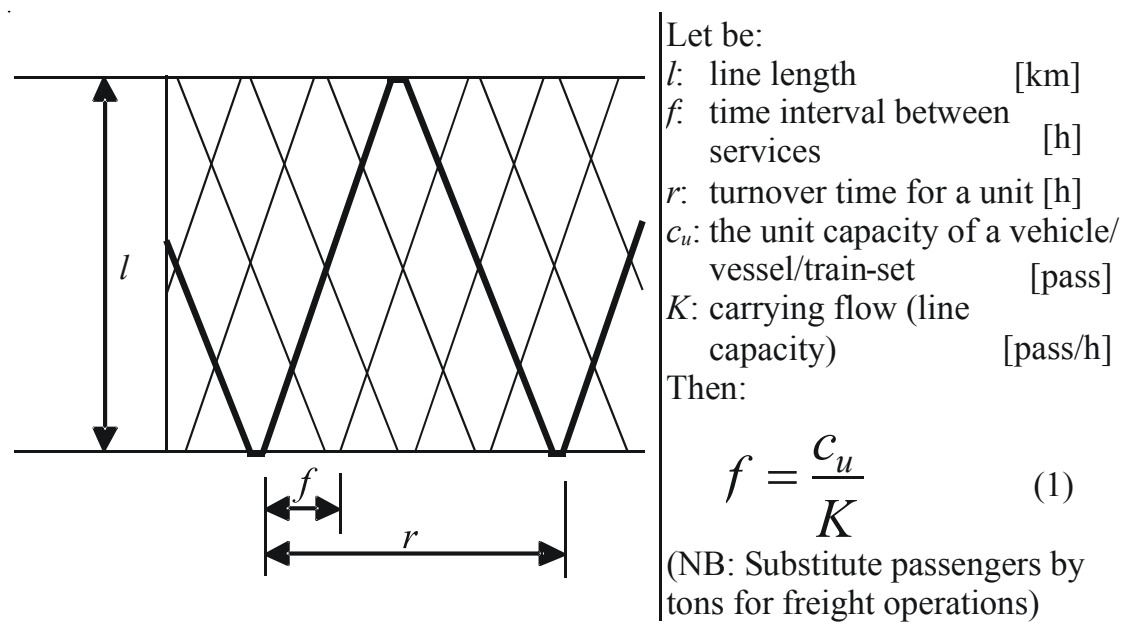

Figure 1: Basic structure of a regular-interval timetable for a shuttle line (space - time diagram) (Source: [7]).

- a common axis of symmetry for all the lines in the network,

- balanced transport supply in opposite directions, with identical travel times,

- scheduled and guaranteed transfers in selected major stations.

\section{Fundamental properties}

There are mainly two readings of Equation (1):

- either $K$ is the actual flow to be carried, and $f$ is the maximum interval between two successive services, derived from the equation;

- or $f$ is the minimum headway, and $K$ is the line theoretical capacity.

Now, let define:

$v_{c} \quad$ as the commercial speed of the service on the line (including turnround time in terminuses)

$n \quad$ as the size of the rolling stock (number of units in operation)

The rolling stock necessary to provide the service can be computed by means of Equation (2), and the turnover time for a unit by means of Equation (3):

$$
n=\frac{r}{f} \quad \text { (2) } \quad r=\frac{2 l}{v_{c}}
$$

By combining the three equations above, we get the fundamental relationship for a shuttle service operated with regular interval as (Equation (4)):

$$
n=2 \frac{K}{v_{c} \cdot c_{u}} l
$$

This equation links the size of the rolling stock, to the unitary capacity of a vehicle/vessel/train-set, the length of the line, the commercial speed, and the transport supply level. 


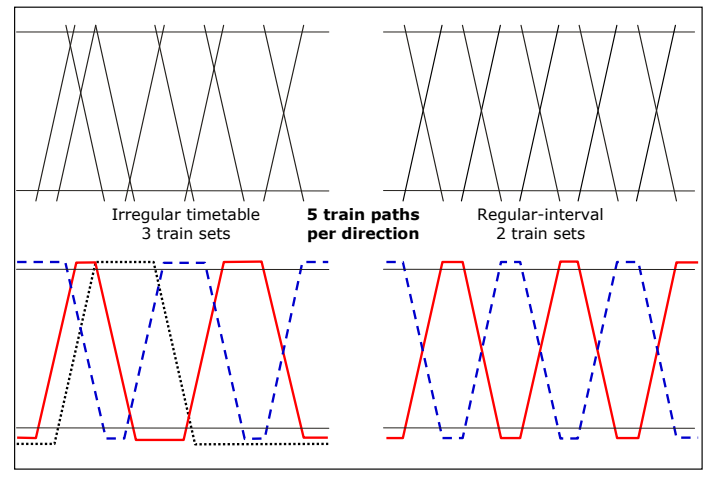

Figure 2: $\quad$ Structuring reduces the need for resources.

Structuring the timetable generates productivity gains, especially when no additional constraints degrade the optimisation (Figure 2). However, setting the interval to any given value (i.e. not linked to the turnover time) may be detrimental to productivity gains - at least partly.

In the case of multiple services running on the same line, each service follows the same regular-interval logic, and the various services are "stacked one upon another", provided that the infrastructure allows for such a superposition. In this case, an extra constraint comes into play: regular intervals should be identical for all services or, at least, modulo between regular intervals should be null (i.e. longer intervals should be a round multiple of the shorter ones, such as - for instance $-30 / 60 / 120$ minutes). This further reduces the optimisation potential. Nevertheless, real-life experience shows that switching to regular- (hourly-) interval operation usually led to eventual gains, sometimes substantial, in resources' productivity.

One of the most interesting properties of regular-interval timetables stems from their periodicity: any particular event is repeating with a period equal to the interval. Therefore, if ever trains meet at any moment in a station, this meeting will occur repeatedly, every hour if the interval is set to 60 minutes. Setting such a meeting in a central node of the network is straightforward: one has just to plan this unique meeting once, by scheduling nearly simultaneous arrivals of all trains in the node, letting enough time for passengers' exchanges, then letting the trains go. The timetable for each line joining the meeting station is wedged in time by means of the arrival/departure times of trains in the central node. If trains running in opposite direction cross at the central node at a given time, and provided that running times are identical for both directions (which is fairly the case in modern networks), crossing of trains will occur at half-the-period time intervals along the line and through time (Figure 3 ). This symmetry propriety can be used to extend the meeting of trains in any station that is distant from the central station to an integer multiple of half the period. If running times make it possible to apply the principle to a triangle of 3 lines, what happens to the central node is exactly repeated to the 2 other nodes of the triangle (Figure 4 ). 


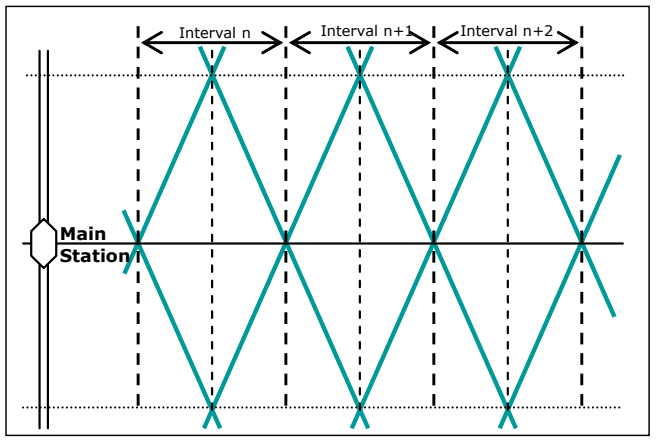

Figure 3: $\quad$ The symmetry propriety.
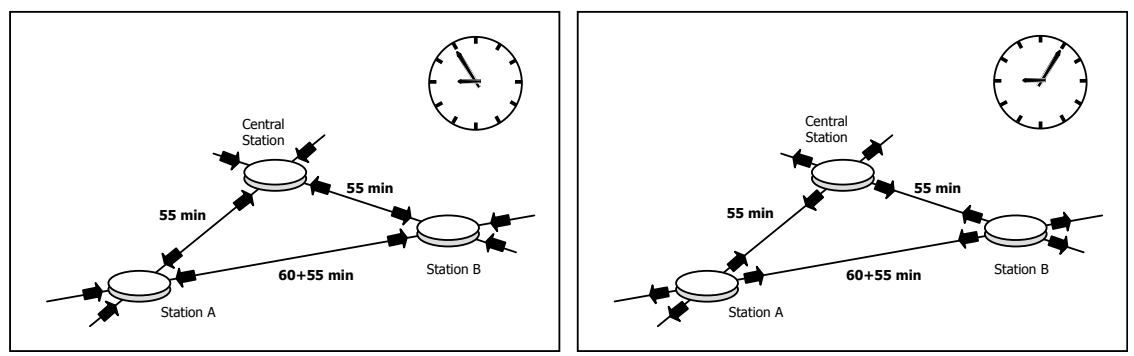

Figure 4: Coordinated 3-nodes network, with link travel times being an integer multiple of the 60 -minutes period.

Thanks to coordination, railway services offer - besides the time coverage provided by the regular operation - spatial coverage. Railway services become available to join any place at any time.

\section{Building elements of a regular-interval timetable}

The first step is to define the fundamental structure of the future transport supply as a more or less abstract set of services, the service backbone (Figure 5).

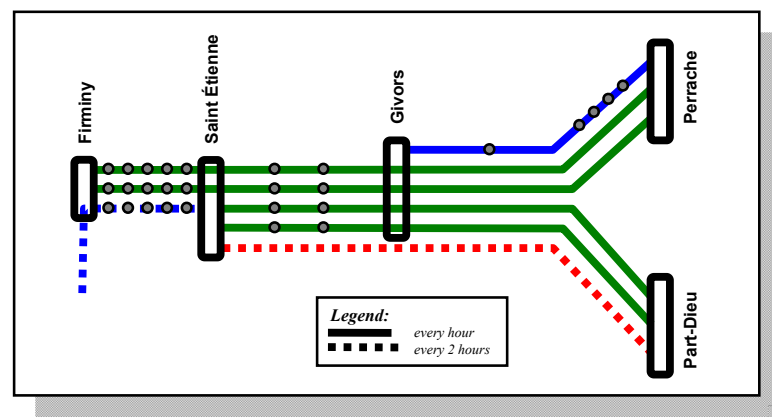

Figure 5: $\quad$ The services backbone (Source: [6]). 


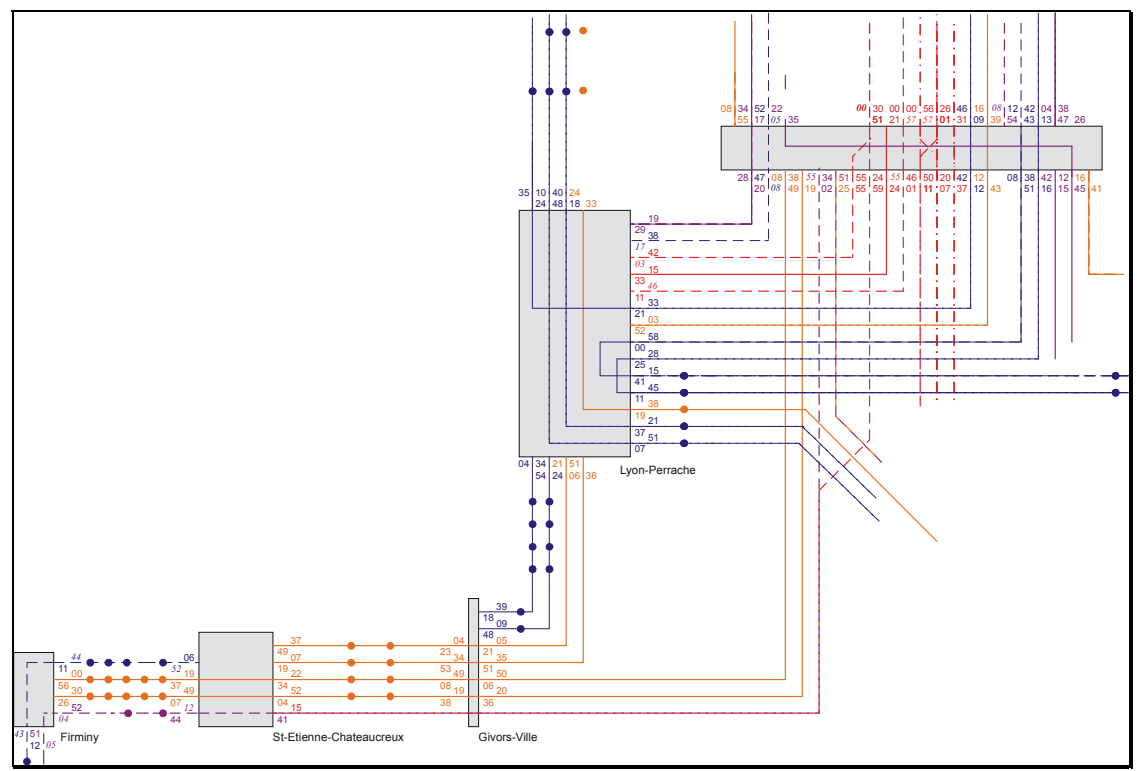

Figure 6: Reticular diagram (Source: [6]).

Designing the basic timetable framework is the second step. This is generally done for a 2-hour time slice and becomes the fundamental raw material used to build the final timetable. Often, the best way to represent the basic framework is a reticular diagram (Figure 6) that shows the network topology. Each line represents a train path able to be repeated every hour, or every two hours.

Next steps involve building the 24-hour timetable for a working day, by repeating the basic framework throughout the day, setting up the early morning and late night services. The whole process is repeated for Sundays and holidays.

\section{Assessment methodology and indicators}

Two main indicators have been developed to capture structural differences among timetables [8]:

- a structure index, reflecting how well the different services comply with the service backbone;

- a regularity index, reflecting how well the final timetable complies with the periodicity defined in the basic framework.

In analysing operational timetables, we may find [8]:

A) Regular train paths belonging to a service, planned at regular time intervals

a) either produced by strictly replicating the train path of the reticular diagram

b) or being "loose" copies of the initial service, i.e. exhibiting slight differences either in travel times or in servicing intermediate stations;

B) Gaps in regularity, i.e. missing train paths that should exist according to the periodicity of the service;

C) Train paths belonging to a service, but planned at irregular time intervals 
a) strictly complying (travel times and stopping patterns) with the definition

b) loosely replicating the initial service;

D) Outliers, i.e. train paths that cannot be traced back to a given service

a) within the normal operational range

b) at the fringe of the operational range, (first and last trains).

Strict compliance with the service backbone or with the regularity (periodicity) is self-explained. To assess loose compliance or not compliance at all, one needs to define tolerance rules. Here is an example of tolerance ranges [8]:

- $[0 \mathrm{~min} ;+4 \mathrm{~min}]$ interval for the departure time at the origin of the service

- $[-4 \mathrm{~min} ;+2 \mathrm{~min}]$ interval for the arrival time at the end station of the service

- no more than 1 extra or less stop in intermediate stations.

In the developed software, users cannot change those rules but are free to set the tolerance thresholds to those that fit best their scope [5].

The assessment methodology is quite sequential. It involves 6 steps [8].

\subsection{Set up a reference reticular diagram}

This will be the reference frame; assessment of compliance will be done by comparing the actual timetable against this reference. The reticular diagram includes implicitly full information on the service backbone, which makes it possible to compute both indexes: structure and regularity. For a given timetable, the underlying reticular diagram may be known or not. In the latter case, some preliminary analysis is needed to reverse-engineer the basic framework out of an existing timetable, which may involve some arbitrary decisions.

\subsection{Set up the tolerance thresholds}

That may be as simple as accepting the default values. Alternately, as already mentioned, users may set their own tolerance thresholds (Figure 7).

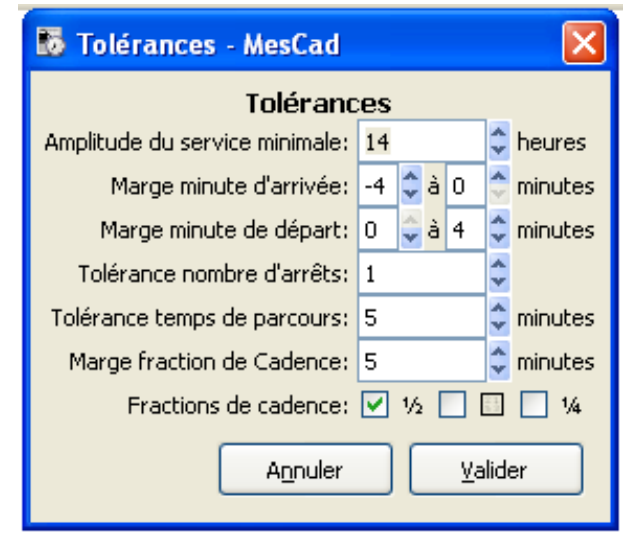

Figure 7: $\quad$ Setting the tolerance thresholds (Source: [5]). 


\subsection{Define the $\mathrm{O} / \mathrm{D}$ relations that will be used in computing the indexes}

This step actually comes to modelling the network as a set of lines. This operation is largely arbitrary and reflects the user's view of the network. Subjectivity, here, is unavoidable. Notwithstanding, experience shows, however, that analysts with fair knowledge of the network come up with pretty close, often identical solutions. Knowledge of the service backbone can help as some diametric lines in the reticular diagram may result from operational concerns and does not necessarily reflect functional objectives. Moreover, users may assign a weight on each line, to take into account volume of demand, or the strategic role of a given line.

\subsection{Define the operational range for each $\mathrm{O} / \mathrm{D}$ relation}

A thumb rule may be that the operational range starts with the first departure of a train path that belongs to a regular-interval planned service, and ends with the last arrival at destination of a train path belonging also to a regular-interval planned service. Implementation for such a rule may be automated, provided that assignment of a train path to a given service is also automated. Alternately, and depending on the design of operations, the operational range may also be based on a fixed number of train paths, or be a fixed time interval, let us say from 6 a.m. to 8 p.m. Ideally, operational range should not be shorter than 13 hours.

\subsection{Assign and label; identify the missing train paths}

For each $\mathrm{O} / \mathrm{D}$ relation and within its operational range, the software assigns to a service every train path and labels it; it also identifies missing train paths within a service as well as outliers. As already seen, there are 4 labels for train paths [8]:

- $\boldsymbol{A}$, train paths belonging to a service planned at regular time intervals

- $\boldsymbol{B}$, missing paths that would exist if a service was planned at regular intervals

- $\boldsymbol{C}$, paths that can be assigned to a service, but not planned at regular intervals

- $\boldsymbol{D}$, outliers that cannot be traced back to a service.

Based on this qualification of train paths, we can define:

- a regularity index as being the ratio

$$
R I=\frac{A}{A+B}
$$

- a structure index as being the ratio

$$
S I=\frac{A+C}{A+C+D}
$$

- and, possibly, a reinforcement rate with the ratio

$$
R R=\frac{C}{A}
$$

Depending on the tolerance thresholds, measured regularity and structure may be strict (with 0 tolerance) or loose (with some tolerance allowed). 


\subsection{Synthesize and display the results for the whole network}

Both regularity and structure indexes are computed for a line and for a service (Figure 8). In what it is proposed, there is already a first aggregation: indexes are computed for the full set of services on a given $\mathrm{O} / \mathrm{D}$ relation.

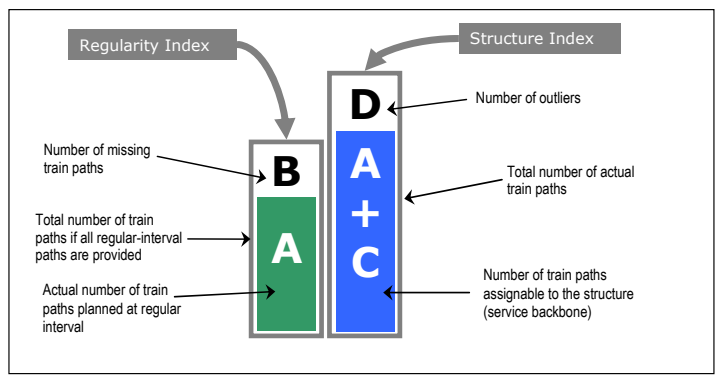

Figure 8: $\quad$ Reading key for the regularity and structure indexes.

The issue of further aggregating the results to build up a unique index for the whole network is still left open. The development team felt that such an additional aggregation will result in unacceptable information loss and that it is actually purposeless. Transport policy makers are sufficiently aware and capable of analysing results on a per line basis; providing a unique performance indicator offers no significant gains in making an overall assessment of the situation.

\section{Limits and drawbacks}

Perfectly regular interval timetables obtain $100 \%$ on both indexes (Figure 9).

By cancelling 2 off-peak train paths (at 10 a.m. and 3 p.m.), the regularity index drops to $86 \%$, but the timetable structure index remains still at $100 \%$.

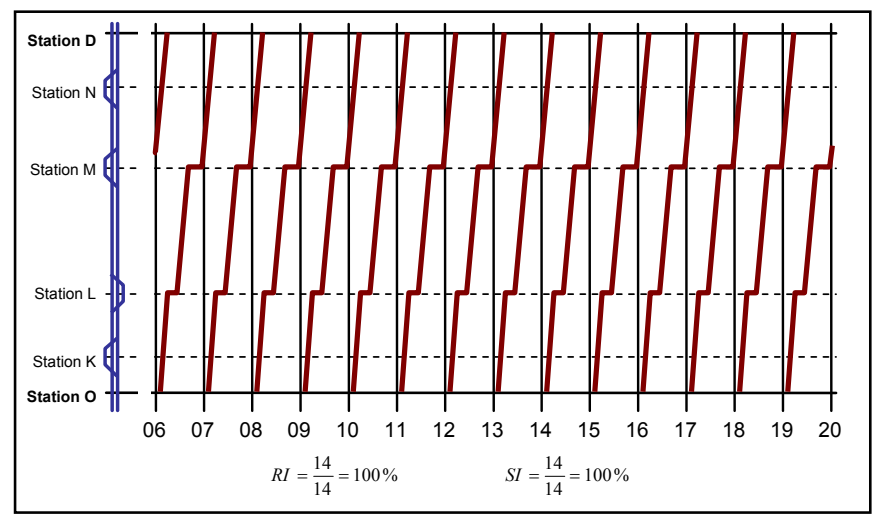

Figure 9: The perfectly complying example (Source: [6]). 


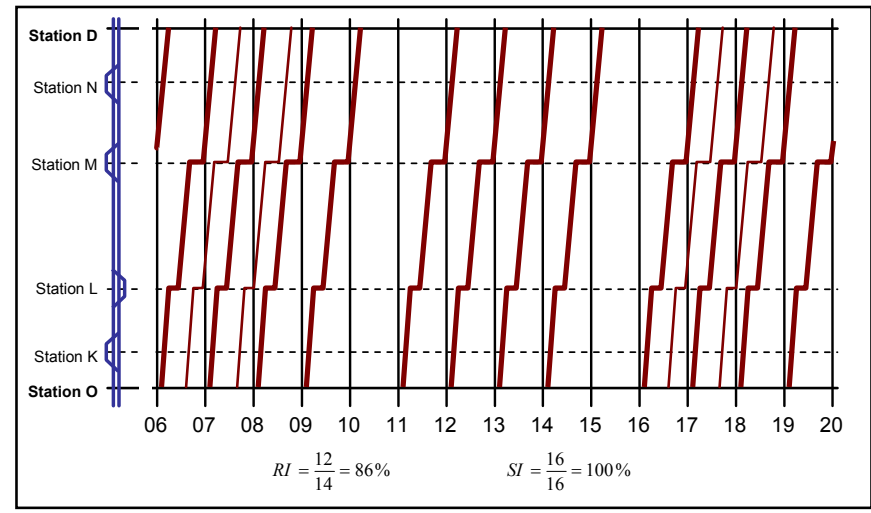

Figure 10: $\quad$ Adding 4 train paths for extra peak services (Source: [6]).

Adding peak-period extra trains (at 6:30, 7:30, 16:30 and 17:30) gives no change in any of those 2 indexes. The reinforcement rate, however, jumps from $0 \%$ to $33 \%$ (Figure 10).

Now, if those 4 extra trains provide additional stops to stations $\mathrm{K}$ and $\mathrm{N}$, they do not comply with the structure anymore and the structure index drops to the $75 \%$ level. This is one of the limits of the methodology. Actually, the 4 extra train paths are identical and can be assigned to a new service; counting them as outliers falsely reduces the structure index. By counting them as a second service, the regularity index drops indeed to $57 \%(12+4$ planned trains for a possible total of $14+14$ train paths), while the structure index remains at $100 \%$.

This issue is related to the arbitrary identification of the services. Preventing it in this particular case is easy enough: one needs only to be systematic in service identification while reverse-engineering the service backbone. The software package does precisely this. However, in most complex cases and with the tolerance thresholds set to non-zero values, the issue is harder to settle, and user's decisions here are critical.

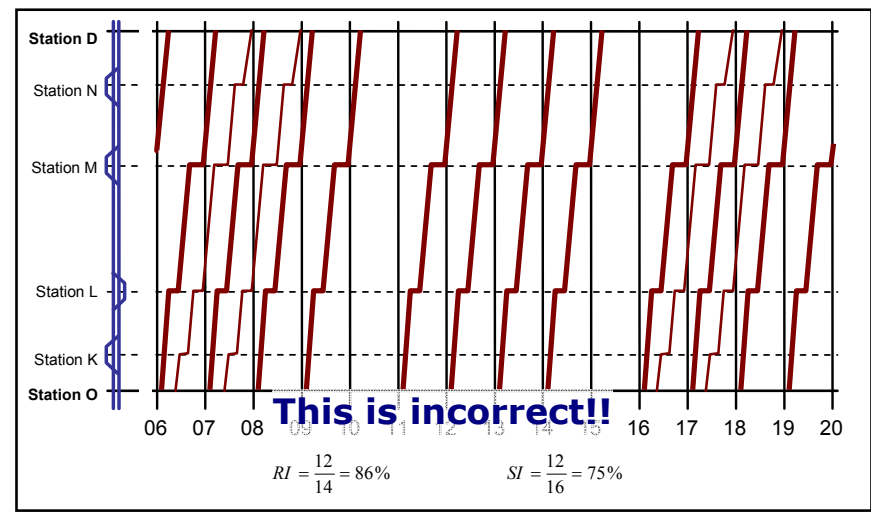

Figure 11: $\quad$ Falsely taking into account the 4 extra trains (Source: [6]). 


\section{Conclusions}

Seamless presence of the rail services is a core objective for a regular-interval timetable. Users should trust the system and be sure that a service is available, all day long, without having to read and decode timetables. Breaches in regularity reduce the system's trustworthiness: customers would need again to consult the timetable before using the services. This raises the need to assess the regularity of actual timetables.

Real life constraints however result in a more-or-less distorted application of the principle and actual timetables often display some irregularities. It is important for the transport authority to assess how well the initial objective of regularity has been achieved in its actual implementation as an operational timetable. Here lies the interest of providing a general methodology to fast and efficiently measure the regularity.

The developed methodology has been eventually implemented in an operational tool [5]. Policy makers can use it to assess the degree of completion of their objectives and, also, to compare alternative timetables. However, the tool reflects the limits of the methodology, which force the user to accept a couple of subjective hypotheses in order to run it. Subjectivity being a part of policy making, having to assume it should not be a major impediment.

\section{References}

[1] Daniel Émery (2009), Mesure du cadencement, Note technique $\mathrm{N}^{\circ} 2$, Retour d'expérience sur la mise en service du cadencement 2008 en RhôneAlpes, EPFL-LITEP, Lausanne (restricted diffusion)

[2] Mohideen Noordeen (1996), Stability analysis of cyclic timetables for a highly interconnected rail network, $\mathrm{PhD}$ Thesis $\mathrm{N}^{\circ} 1435$, EPFL, Lausanne

[3] Werner Stohler (2003), Why is an integrated clockface-driven railway system more efficient than a divided competition-oriented railway system? SMA und Partner AG, Zürich

[4] Werner Stohler (1993), La planification de la gestion et de l'exploitation ferroviaire, in Rail International, Paris, 10/1993; pp. 64-70

[5] David Tron, Panos Tzieropoulos (2009), How regular is a regular-interval timetable? An operational tool to assess regularity, Swiss Transport Research Conference STRC 09, Monte Veritá, Ascona

[6] Panos Tzieropoulos, Daniel Émery (2009), De la théorie à la pratique, in Préconisations, Retour d'expérience sur la mise en service du cadencement 2008 en Rhône-Alpes, EPFL-LITEP, Lausanne (restricted diffusion)

[7] Panos Tzieropoulos, Daniel Émery, Jean-Daniel Buri (2009), Regularinterval timetables; Theoretical foundations and policy implications, presented in the $12^{\text {th }}$ World Conference on Transportation Research, Lisbon

[8] Panos Tzieropoulos et al (2008), Qualité du cadencement, in Diagnostic, Retour d'expérience sur la mise en service du cadencement 2008 en RhôneAlpes, EPFL-LITEP, Lausanne (restricted diffusion) 